Proyecciones Journal of Mathematics

Vol. 32, No 3, pp. 235-244, September 2013.

Universidad Católica del Norte

Antofagasta - Chile

\title{
Some results on global dominating sets
}

\author{
S. K. Vaidya \\ Saurashtra University, INDIA \\ and \\ R. M. Pandit \\ A. V. Parekh Technical Institute, INDIA \\ Received: June 2012. Accepted : May 2013
}

\begin{abstract}
A dominating set is called a global dominating set if it is a dominating set for a graph $G$ and its complement $\bar{G}$. We investigate some general results for global dominating sets corresponding to the graphs $P_{n}, C_{n}$ and $W_{n}$.
\end{abstract}

Keywords : Duplication of a vertex, dominating set, global dominating set.

AMS subject classification number : 05C69; 05C75. 


\section{Introduction}

We begin with finite connected and undirected graph $G=(V, E)$ without loops and multiple edges. The set $S \subseteq V$ of vertices in a graph $G$ is called a dominating set if every vertex $v \in V$ is either an element of $S$ or is adjacent to an element of $S$. A dominating set $S$ is a minimal dominating $\operatorname{set}(\mathrm{MDS})$ if no proper subset $S^{\prime} \subset S$ is a dominating set.

The minimum cardinality of a dominating set of $G$ is called a domination number of $G$ which is denoted by $\gamma(G)$ and the corresponding dominating set is called a $\gamma$-set of $G$.

The open neighborhood $N(v)$ of $v \in V$ is the set of vertices adjacent to $v$ and the closed neighborhood of $v$ is the set $N[v]=N(v) \cup\{v\}$.

The complement $\bar{G}$ of $G$ is the graph with vertex set $V$ and two vertices are adjacent in $\bar{G}$ if and only if they are not adjacent in $G$.

A subset $D \subseteq V$ is called a global dominating set in $G$ if $D$ is a dominating set of both $G$ and $\bar{G}$. The global domination number $\gamma_{g}(G)$ is the minimum cardinality of a minimal global dominating set in $G$. The concept of global domination in graph was introduced by Sampathkumar [4].

The wheel graph $W_{n}$ is defined to be the join $C_{n-1}+K_{1}$. The vertex corresponding to $K_{1}$ is known as apex vertex and the vertices corresponding to cycle are known as rim vertices.

Duplication of a vertex $v$ of a graph $G$ produces a new graph $G^{\prime}$ by adding a vertex $v^{\prime}$ with $N\left(v^{\prime}\right)=N(v)$. In other words a vertex $v^{\prime}$ is said to be duplication of $v$ if all the vertices which are adjacent to $v$ are now adjacent to $v^{\prime}$ also.

If the vertices of a graph $G$ are duplicated altogether then the resultant graph is known as splitting graph of $G$ which is denoted as $S^{\prime}(G)$.

For all standard terminology and notation in graph theory we refer to West [6] while for terminology related to domination in graphs we rely upon Haynes et al. [2]. The various bounds of global domination number in terms of order, degrees and domination number of graph is given by Brigham and Duttom [1]. The global domination number for one-point union of finite number of copies of cycles $C_{n}$ is reported in Vaidya and Pandit [5] while Kulli and Janakiram [3] have introduced the concept of total global dominating sets.

In the present work we investigate some general results which relate the concept of global domination and duplication of a vertex. 


\section{Some General Results}

Lemma 2.1. For cycle $C_{n}$, let $C_{n}^{\prime}$ be the graph obtained by duplication of a vertex $x$ by $x^{\prime}$ where $x \in V\left(C_{n}\right)$. If $S$ is a dominating set of $C_{n}$ containing either of the vertices which are adjacent to $x$, then $S$ is also a dominating set of $C_{n}^{\prime}$.

Proof: If $x \in V\left(C_{n}\right)$ is duplicated by a vertex $x^{\prime}$ then $V\left(C_{n}^{\prime}\right)=V\left(C_{n}\right) \cup$ $\left\{x^{\prime}\right\}$. Now if $S$ is a dominating set of $C_{n}$ and $a \in S(a \neq x)$ dominates $x$ then $a$ is adjacent to $x^{\prime}$ in $C_{n}^{\prime}$. Thus, $a$ dominates $x^{\prime}$ in $C_{n}^{\prime}$. That is, $S$ is a dominating set of $C_{n}^{\prime}$.

Theorem 2.2. Let $C_{n}^{\prime}$ be the graph obtained by duplication of a vertex $x$ of $C_{n}$ by $x^{\prime}$. If $S$ is a global dominating set of $C_{n}$ containing either of the vertices which are adjacent to $x$ then $S$ is also a global dominating set of $C_{n}^{\prime}$.

Proof: If $S$ is a global dominating set of $C_{n}$ then $S$ is a dominating set of $C_{n}$ as well as $\overline{C_{n}}$. As $S$ is a dominating set of $C_{n}$ then according to Lemma 2.1, $S$ is also a dominating set of $C_{n}^{\prime}$.

To prove the required result it remains to show that $S$ is a dominating set of $\overline{C_{n}^{\prime}}$. For that if $a \in S$ is adjacent to $x$ in $C_{n}^{\prime}$ then it is not adjacent to $x$ in $\overline{C_{n}^{\prime}}$. Now, $S$ being a dominating set of $\overline{C_{n}} \exists$ a vertex $b \in S, a \neq b$ such that $b$ is not adjacent to $x$ in $C_{n}$ but dominates $x$ in $\overline{C_{n}}$. As $a \in S$ is adjacent to both $x$ and $x^{\prime}$ in $C_{n}^{\prime}$ implies that it is not adjacent to both $x$ and $x^{\prime}$ in $\overline{C_{n}^{\prime}}$. Moreover, $V\left(\overline{C_{n}^{\prime}}\right)=V\left(\overline{C_{n}}\right) \cup\left\{x^{\prime}\right\}$ and $S$ is a dominating set of $\overline{C_{n}}$ then above referred vertex $b \in S$ must dominate $x^{\prime}$ in $\overline{C_{n}^{\prime}}$. Hence, $S$ is also a dominating set of $\overline{C_{n}^{\prime}}$.

Thus, $S$ is a dominating set of both $C_{n}^{\prime}$ as well as $\overline{C_{n}^{\prime}}$. Therefore, $S$ is a global dominating set of $C_{n}^{\prime}$.

Theorem 2.3. If $S$ is a $\gamma$-set of $P_{n}(n \geq 6)$ then $S$ is a global dominating set of $P_{n}$. Also $\gamma\left(P_{n}\right)=\gamma_{g}\left(P_{n}\right)$.

Proof: For $P_{n}, n \geq 6$, consider a $\gamma$-set, $S=\left\{v_{2}, v_{5}, \ldots, v_{3 j+2}\right\}$ if $n \equiv$ 0 or $2(\bmod 3)$ and $S=\left\{v_{2}, v_{5}, \ldots, v_{3 j+2}\right\} \cup\left\{v_{n-1}\right\}$ if $n \equiv 1(\bmod 3)$ where $0 \leq j \leq\left\lfloor\frac{n-2}{3}\right\rfloor$.

In $P_{n}$, there are two vertices of degree 1 and $(n-2)$ internal vertices are of degree 2. Now, let $v_{i} \in S$ and $v_{j} \in S$ be any two vertices such that $v_{j} \notin N\left[v_{i}\right]$. 
Then we claim that these two vertices are sufficient to dominate remaining vertices of $\overline{P_{n}}$. Since the vertices which are not in $N\left(v_{i}\right)$ must belong to $N\left(v_{j}\right)$, any $S \subset V$ containing $v_{i}$ and $v_{j}$ will be a dominating set of $\overline{P_{n}}$. Thus, $S$ is a dominating set of both $P_{n}$ as well as $\overline{P_{n}}$. Hence, $S$ is a global dominating set of $P_{n}$.

Since, $S$ being a $\gamma$-set, it is of minimum cardinality. Therefore, $\gamma\left(P_{n}\right)=$ $\gamma_{g}\left(P_{n}\right)$ for $n \geq 6$.

\section{Remark 2.4.}

(i) $S=\left\{v_{2}\right\}$ is a $\gamma$-set of both $P_{2}$ and $P_{3}$ which is not a dominating set of $\overline{P_{2}}$ as well as of $\overline{P_{3}}$ and hence it is not a global dominating set of $P_{2}$ as well as of $P_{3}$. Also $\gamma\left(P_{n}\right) \neq \gamma_{g}\left(P_{n}\right)$ for $n=2,3$ as $\gamma\left(P_{2}\right)=\gamma\left(P_{3}\right)=1 \neq$ $2=\gamma_{g}\left(P_{2}\right)=\gamma_{g}\left(P_{3}\right)$.

(ii) $S=\left\{v_{1}, v_{4}\right\}$ is a $\gamma$-set as well as a global dominating set of $P_{4}$ while $S=\left\{v_{1}, v_{3}\right\}$ and $S=\left\{v_{2}, v_{4}\right\}$ are such $\gamma$-sets of $P_{4}$ which are not dominating sets of $\overline{P_{4}}$ and hence they are not global dominating sets of $P_{4}$. But $\gamma\left(P_{4}\right)=\gamma_{g}\left(P_{4}\right)=2$.

(iii) $S=\left\{v_{2}, v_{5}\right\}$ is a $\gamma$-set as well as a global dominating set of $P_{5}$ while $S=\left\{v_{2}, v_{4}\right\}$ is such a $\gamma$-set of $P_{5}$ which is not a dominating set of $\overline{P_{5}}$ and hence it is not a global dominating set of $P_{5}$. But $\gamma\left(P_{5}\right)=\gamma_{g}\left(P_{5}\right)=2$.

Therefore, $\gamma$-set of $P_{n}(n \leq 5)$ may or may not be a global dominating set of $P_{n}$.

Theorem 2.5. $S$ is a global dominating set of $P_{n}$ if and only if it is a global dominating set of $P_{n}^{\prime}$.

Proof: For $P_{n}(n \geq 4)$, consider the global dominating set $S=\left\{v_{2}, v_{5}, v_{8}, \ldots\right.$, $\left.v_{3 j+2}\right\}$ if $n \equiv 0$ or $2(\bmod 3)$ and $S=\left\{v_{2}, v_{5}, v_{8}, \ldots, v_{3 j+2}\right\} \cup\left\{v_{n-1}\right\}$ if $n \equiv 1(\bmod 3)$ where $0 \leq j \leq\left\lfloor\frac{n-2}{3}\right\rfloor$.

There are three possibilities when duplication of a vertex of $P_{n}$ takes place.

(i) Duplication of a pendant vertex.

(ii) Duplication of an internal vertex not belonging to $S$ 
(iii) Duplication of an internal vertex belonging to $S$.

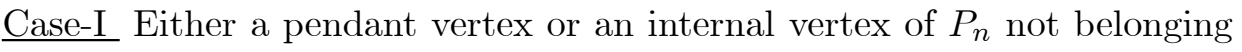
to $S$ is duplicated.

Here, a duplicated vertex $v^{\prime}$ is adjacent to a vertex in above referred $S$ and $V\left(P_{n}^{\prime}\right)=V\left(P_{n}\right) \cup\left\{v^{\prime}\right\}$.

Hence, $S$ is a global dominating set of $P_{n}^{\prime}$.

Case-II An internal vertex of $P_{n}$ belonging to $S$ is duplicated.

For $P_{n}(n \geq 4)$, consider the global dominating set $S=\left\{v_{1}, v_{4}, v_{7}, \ldots, v_{3 j+1}\right\}$

if $n \equiv 1 \operatorname{or} 2(\bmod 3)$ and $S=\left\{v_{1}, v_{4}, v_{7}, \ldots, v_{3 j+1}\right\} \cup\left\{v_{n}\right\}$ if $n \equiv 0(\bmod 3)$ where $0 \leq j \leq\left\lfloor\frac{n-1}{3}\right\rfloor$.

Here, the duplicated vertex $v^{\prime}$ is adjacent to a vertex in above referred $S$ and $V\left(P_{n}^{\prime}\right)=V\left(P_{n}\right) \cup\left\{v^{\prime}\right\}$. Hence, $S$ is a global dominating set of $P_{n}^{\prime}$.

Conversely, suppose that $S$ is a global dominating set of $P_{n}^{\prime}$. Therefore, $S$ is a dominating set of both $P_{n}^{\prime}$ and $\overline{P_{n}^{\prime}}$. But $V\left(P_{n}^{\prime}\right)=V\left(P_{n}\right) \cup\left\{v^{\prime}\right\}$ and $V\left(\overline{P_{n}^{\prime}}\right)=V\left(\overline{P_{n}}\right) \cup\left\{v^{\prime}\right\}$. Moreover, $S$ being a global dominating set of $P_{n}^{\prime} \exists$ a vertex in $S$ which will dominate both the vertices $v$ and $v^{\prime}$ in $P_{n}^{\prime}$ as well as in $\overline{P_{n}^{\prime}}$. Hence, $S$ is a dominating set of both $P_{n}$ and $\overline{P_{n}}$. Therefore, $S$ is a global dominating set of $P_{n}$.

Hence, we have proved that $S$ is a global dominating set of $P_{n}$ if and only if it is a global dominating set of $P_{n}^{\prime}$.

Theorem 2.6. $\gamma_{g}\left(P_{n}^{\prime}\right)=\gamma_{g}\left(P_{n}\right)$ for $n=2,3$.

Proof: For path $P_{2}, V\left(P_{2}\right)=\left\{v_{1}, v_{2}\right\}$. Then $S=\left\{v_{1}, v_{2}\right\}$ is a global dominating set of $P_{2}$ which is also an MDS with minimum cardinality. Hence, $\gamma_{g}\left(P_{2}\right)=2$.

Now on duplicating either of the pendant vertices $v$ of $P_{2}$ by a vertex $v^{\prime}, S=\left\{v_{1}, v_{2}\right\}$ is a global dominating set of $P_{2}^{\prime}$ which is also an MDS with minimum cardinality. Hence, $\gamma_{g}\left(P_{2}^{\prime}\right)=2$.

Thus, $\gamma_{g}\left(P_{2}^{\prime}\right)=\gamma_{g}\left(P_{2}\right)$.

Next, consider the path $P_{3}, V\left(P_{3}\right)=\left\{v_{1}, v_{2}, v_{3}\right\}$. Then $S=\left\{v_{1}, v_{2}\right\}$ is a global dominating set of $P_{3}$ which is also an MDS with minimum cardinality. Hence, $\gamma_{g}\left(P_{3}\right)=2$.

Here, duplication of a vertex can take place in either of the following manner: 
$\underline{\text { Case-I }}$

If $v^{\prime}$ is the duplicated vertex of either of the pendant vertices of $P_{3}$ then $S=\left\{v_{1}, v_{2}\right\}$ is a global dominating set of $P_{3}^{\prime}$ which is also an MDS with minimum cardinality. Hence, $\gamma_{g}\left(P_{3}^{\prime}\right)=2$.

$\underline{\text { Case-II }}$

If $v^{\prime}$ is the duplicated vertex of an internal vertex $v$ of $P_{3}$ then $S=$ $\left\{v_{1}, v_{2}\right\}$ is a global dominating set of $P_{3}^{\prime}$ which is also an MDS with minimum cardinality. Hence, $\gamma_{g}\left(P_{3}^{\prime}\right)=2$.

Thus, $\gamma_{g}\left(P_{3}^{\prime}\right)=\gamma_{g}\left(P_{3}\right)$.

Hence, we have proved that $\gamma_{g}\left(P_{n}^{\prime}\right)=\gamma_{g}\left(P_{n}\right)$ for $n=2,3$.

Theorem 2.7. For $n \geq 4$,

$$
\gamma_{g}\left(P_{n}^{\prime}\right)=\gamma_{g}\left(P_{n}\right) \text { for } n \equiv 1 \text { or } 2(\bmod 3)
$$

and

$$
\left.\begin{array}{rlrl}
\gamma_{g}\left(P_{n}{ }^{\prime}\right) & =\gamma_{g}\left(P_{n}\right) ; & & \text { if } v_{i} \notin S \\
& =\gamma_{g}\left(P_{n}\right)+1 ; & & \text { if } v_{i} \in S
\end{array}\right\} \text { for } n \equiv 0(\bmod 3)
$$

where $v_{i}$ is the vertex of $P_{n}$ which is to be duplicated.

Proof: For $P_{n}(n \geq 4)$, consider the global dominating set $S=\left\{v_{2}, v_{5}, v_{8}, \ldots\right.$, $\left.v_{3 j+2}\right\}$ if $n \equiv 0$ or $2(\bmod 3)$ and $S=\left\{v_{2}, v_{5}, v_{8}, \ldots, v_{3 j+2}\right\} \cup\left\{v_{n-1}\right\}$ if $n \equiv 1(\bmod 3)$ where $0 \leq j \leq\left\lfloor\frac{n-2}{3}\right\rfloor$.

Consider $S=\left\{v_{2}, v_{5}, v_{8}, \ldots, v_{3 j+2}\right\}$ if $n \equiv 0(\bmod 3)$. Then, for $v_{i} \in S$, $S-\left\{v_{i}\right\}$ will not be a dominating set as the vertices $v_{i-1}$ and $v_{i+1}$ will not be dominated by any of the vertices of $S-\left\{v_{i}\right\}$. Hence, $S$ is an MDS of $P_{n}$.

For $n \equiv 0(\bmod 3),|S|=\frac{n}{3}$. Suppose, if possible, $S_{1} \neq S$ is an MDS with $\left|S_{1}\right|=\frac{n}{3}-1<|S|$. Any path $P_{n}$ has total $n$ vertices and any vertex $v$ of $P_{n}$ dominates either 1 or 2 vertices of $P_{n}$ other than vertex $v$. Therefore, $\left(\frac{n}{3}-1\right)$ vertices of $S_{1}$ cannot dominate more than $\left|S_{1}\right|+2\left|S_{1}\right|$ vertices. But $\left|S_{1}\right|+2\left|S_{1}\right|=\left(\frac{n}{3}-1\right)+2\left(\frac{n}{3}-1\right)=\frac{n}{3}-1+\frac{2 n}{3}-2=\frac{3 n}{3}-3=n-3<n=\mid V\left(P_{n} \mid\right.$. Hence, $S_{1}$ is not a dominating set of $P_{n}$, which is a contradiction.

Therefore, $S$ is an MDS with minimum cardinality.

$\underline{\text { Case-I }} n \equiv 1(\bmod 3)$. 
$\underline{\text { Subcase-1 }}$ When a pendant vertex or an internal vertex of $P_{n}$ not belonging to $S$ is duplicated.

Here, $S=\left\{v_{2}, v_{5}, v_{8}, \ldots, v_{3 j+2}\right\} \cup\left\{v_{n-1}\right\}$ is a global dominating set of $P_{n}$ as well as of $P_{n}^{\prime}$ as we have proved in Theorem 2.5 and it is an MDS of minimum cardinality as proved above. Therefore, $\gamma_{g}\left(P_{n}^{\prime}\right)=\gamma_{g}\left(P_{n}\right)$ for $n \equiv 1(\bmod 3)$.

Subcase-2 An internal vertex of $P_{n}$ belonging to $S$ is duplicated.

Here, $S=\left\{v_{1}, v_{4}, v_{7}, \ldots, v_{3 j+1}\right\}$ is a global dominating set of both $P_{n}^{\prime}$ and $P_{n}$ as we have proved in Theorem 2.5 and it is an MDS of minimum cardinality as proved above. Therefore, $\gamma_{g}\left(P_{n}^{\prime}\right)=\gamma_{g}\left(P_{n}\right)$ for $n \equiv 1(\bmod 3)$. Further, we have the following cases :

$\underline{\text { Case- } 2} n \equiv 2(\bmod 3)$.

$\underline{\text { Subcase-1 }}$ When a pendant vertex or an internal vertex of $P_{n}$ not belonging to $S$ is duplicated.

$S=\left\{v_{2}, v_{5}, v_{8}, \ldots, v_{3 j+2}\right\}$ if $n \equiv 2(\bmod 3)$.

Subcase-2 An internal vertex of $P_{n}$ belonging to $S$ is duplicated.

$S=\left\{v_{1}, v_{4}, v_{7}, \ldots, v_{3 j+1}\right\}$ if $n \equiv 2(\bmod 3)$.

$\underline{\text { Case- } 3} n \equiv 0(\bmod 3)$.

$\underline{\text { Subcase-1 }}$ When a pendant vertex or an internal vertex of $P_{n}$ not belonging to $S$ is duplicated.

$S=\left\{v_{2}, v_{5}, v_{8}, \ldots, v_{3 j+2}\right\}$ if $n \equiv 0(\bmod 3)$.

We treat the Subcase-2 of Case-3 separately.

Subcase-2 An internal vertex of $P_{n}$ belonging to $S$ is duplicated.

Here, $S=\left\{v_{1}, v_{4}, v_{7}, \ldots, v_{3 j+1}\right\} \cup\left\{v_{n}\right\}$ and $S=\left\{v_{2}, v_{5}, v_{8}, \ldots, v_{3 j+2}\right\}$ are global dominating sets of $P_{n}^{\prime}$ and $P_{n}$ respectively.

Now, the respective sets $S$ are global dominating sets of $P_{n}$ as well as of $P_{n}^{\prime}$ as we have proved in Theorem 2.5 and following the analogous argument as above, it can be shown that the respective sets $S$ are MDS with 
minimum cardinality.

Thus, for $n \geq 4$,

$$
\gamma_{g}\left(P_{n}^{\prime}\right)=\gamma_{g}\left(P_{n}\right) \text { for } n \equiv 1 \operatorname{or} 2(\bmod 3)
$$

and

$$
\left.\begin{array}{rlrl}
\gamma_{g}\left(P_{n}^{\prime}\right) & =\gamma_{g}\left(P_{n}\right) ; & & \text { if } v_{i} \notin S \\
& =\gamma_{g}\left(P_{n}\right)+1 ; & & \text { if } v_{i} \in S
\end{array}\right\} \text { for } n \equiv 0(\bmod 3)
$$

where $v_{i}$ is the vertex of $P_{n}$ which is to be duplicated.

Theorem 2.8. $S$ is a global dominating set of $W_{n}(n \geq 4)$ if and only if it is a global dominating set of $S^{\prime}\left(W_{n}\right)$.

Proof: Consider the wheel $W_{n}=C_{n-1}+K_{1}$ with $v_{1}, v_{2}, v_{3}, \ldots, v_{n}$ as its rim vertices and $c_{1}$ as its apex vertex.

Let $v_{1}{ }^{\prime}, v_{2}{ }^{\prime}, v_{3}{ }^{\prime}, \ldots, v_{n}{ }^{\prime}$ be the duplicated vertices corresponding to $v_{1}, v_{2}, v_{3}$, $\ldots, v_{n}$ while $c_{1}{ }^{\prime}$ be the duplication of $c_{1}$.

We observe that $S=\left\{v_{1}, v_{2}, v_{3}, c_{1}\right\}$ is the global dominating set of $W_{4}$ as well as $S^{\prime}\left(W_{4}\right)$.

For $n \geq 5$, consider a global dominating set of $W_{n}, S=\left\{v_{1}, v_{2}, c_{1}\right\}$. Now, $V\left(S^{\prime}\left(W_{n}\right)\right)=V\left(W_{n}\right) \cup V\left(W_{n}^{d}\right)$ where $V\left(W_{n}^{d}\right)=\left\{v_{1}{ }^{\prime}, v_{2}{ }^{\prime}, v_{3}{ }^{\prime}, \ldots, v_{n}{ }^{\prime}, c_{1}^{\prime}\right\}$.

From the definition of splitting graph, any vertex in $S$ which dominates $v \in V\left(S^{\prime}\left(W_{n}\right)\right)$ will also dominate the duplicated vertex $v^{\prime}$ of $v$ in $S^{\prime}\left(W_{n}\right)$. Hence, $S$ being a dominating set of $W_{n}$, it is also a dominating set of $S^{\prime}\left(W_{n}\right)$.

Now, it remains to show that $S$ is a dominating set of $\overline{S^{\prime}\left(W_{n}\right)}$.

From the definition of duplication of a vertex, it is obvious that

(i) a vertex $v$ and its duplicated vertex $v^{\prime}$ can never be adjacent in $S^{\prime}\left(W_{n}\right)$. Therefore, they are adjacent in $\overline{S^{\prime}\left(W_{n}\right)}$.

(ii) any vertex in $S$ which dominates $v$ will also dominate $v^{\prime}$ in $\overline{S^{\prime}\left(W_{n}\right)}$ and $S$ is a dominating set of $W_{n}$ implies that $S$ is a dominating set of $\overline{S^{\prime}\left(W_{n}\right)}$.

Thus, $S$ is a dominating set of both $S^{\prime}\left(W_{n}\right)$ and $\overline{S^{\prime}\left(W_{n}\right)}$. Therefore, $S$ is a global dominating set of $S^{\prime}\left(W_{n}\right)$.

Conversely, suppose that $S$ is a global dominating set of $S^{\prime}\left(W_{n}\right)$. Then, $S$ is a dominating set of both $S^{\prime}\left(W_{n}\right)$ and $\overline{S^{\prime}\left(W_{n}\right)}$. But $V\left(S^{\prime}\left(W_{n}\right)\right)=$ $V\left(W_{n}\right) \cup V\left(W_{n}^{d}\right)$ and $V\left(\overline{S^{\prime}\left(W_{n}\right)}\right)=V\left(\overline{W_{n}}\right) \cup V\left(W_{n}^{d}\right)$ which implies that $S$ is a dominating set of both $W_{n}$ and $\overline{W_{n}}$. Hence, $S$ is a global dominating set of $W_{n}$. 
Thus, we have proved that $S$ is a global dominating set of $W_{n}$ if and only if it is a global dominating set of $S^{\prime}\left(W_{n}\right)$.

\section{Concluding Remarks}

Some structural properties are derived corresponding to the concept of global dominating sets. Analogous results can be obtained for other graph families and in the context of various types of dominating sets in graphs.

\section{Acknowledgement}

The authors are highly thankful to the anonymous referee for careful reading of the first draft of this paper.

\section{References}

[1] R. C. Brigham and R. D. Dutton, Factor domination in graphs, Discrete Mathematics, 86, pp. 127-136, (1990).

[2] T. W. Haynes, S. T. Hedetniemi and P. J. Slater, Fundamentals of Domination in Graphs, Monographs and Textbooks in Pure and Applied Mathematics, Marcel Dekker, Inc., New York, (1998).

[3] V. R. Kulli and B. Janakiram, The total global domination number of a graph, Indian Journal of Pure and Applied Mathematics, 27, pp. 537-542, (1996).

[4] E. Sampathkumar, The global domination number of a graph, J. Math. Phys.Sci., 23, pp. 377-385, (1989).

[5] S. K. Vaidya and R. M. Pandit, Some new results on global dominating sets, ISRN Discrete Mathematics,vol. 2012, Article ID 852129, 6 pages, 2012. doi: $10.5402 / 2012 / 852129$.

[6] D. B. West, Introduction to Graph Theory, Prentice-Hall of India, New Delhi, (2003). 


\section{S. K. Vaidya}

Department of Mathematics

Saurashtra University

Rajkot - 360005

Gujarat

INDIA

e-mail : samirkvaidya@yahoo.co.in

and

R. M. Pandit

Department of Mathematics

A. V. Parekh Technical Institute, Rajkot - 360002

Gujarat

INDIA

e-mail : pandit.rajesh@ymail.com 